\title{
Traumatic spondyloptosis: a series of 20 patients
}

\author{
Akash Mishra, MS, Deepak Agrawal, MCh, Deepak Gupta, MCh, Sumit Sinha, MCh, \\ Guru D. Satyarthee, MCh, and Pankaj K. Singh, MCh
}

Department of Neurosurgery, Jai Prakash Narayan Apex Trauma Center, All India Institute of Medical Sciences, New Delhi, India

\begin{abstract}
OBJECT Spondyloptosis represents the most severe form of spondylolisthesis, which usually follows high-energy trauma. Few reports exist on this specific condition, and the largest series published to date consists of only 5 patients. In the present study the authors report the clinical observations and outcomes in a cohort of 20 patients admitted to a regional trauma center for severe injuries including spondyloptosis.
\end{abstract}

METHODS The authors performed a retrospective chart review of patients admitted with spondyloptosis at their department over a 5-year period (March 2008-March 2013). Clinical, radiological, and operative details were reviewed for all patients.

RESULTS In total, 20 patients with spondyloptosis were treated during the period reviewed. The mean age of the patients was 27 years (range 12-45 years), and 17 patients were male ( 2 boys and 15 men) and 3 were women. Fall from height (45\%) and road traffic accidents (35\%) were the most common causes of the spinal injuries. The grading of the American Spinal Injury Association (ASIA) was used to assess the severity of spinal cord injury, which for all patients was ASIA Grade A at the time of admission. In 11 patients (55\%), the thoracolumbar junction (T10-L2) was involved in the injury, followed by the dorsal region (T1-9) in 7 patients (35\%); 1 patient (5\%) had lumbar and 1 patient $(5 \%)$ sacral spondyloptosis. In 19 patients (95\%), spondyloptosis was treated surgically, involving the posterior route in all cases. In 7 patients (37\%), corpectomy was performed. None of the patients showed improvement in neurological deficits. The mean follow-up length was 37.5 months (range 3-60 months), and 5 patients died in the follow-up period from complications due to formation of bedsores (decubitus ulcers).

CONCLUSIONS To the authors' best knowledge, this study was the largest of its kind on traumatic spondyloptosis. Its results illustrate the challenges of treating patients with this condition. Despite deformity correction of the spine and early mobilization of patients, traumatic spondyloptosis led to high morbidity and mortality rates because the patients lacked access to rehabilitation facilities postoperatively.

http://thejns.org/doi/abs/10.3171/2014.10.SPINE1440

KEY WORDS spondyloptosis; spinal cord injury; TLICS score; ASIA; complication; surgery; spinal disorders; trauma

$\mathrm{S}$ PINAL cord injuries, although on a decline in developed countries, continue to have a high incidence in developing countries such as India. At our center, we received 520 cases of spinal injury in the year 2012 alone. Of these, 289 were injuries of the cervical spine, 112 of the thoracic spine, and 119 of the lumbar spine, highlighting the magnitude of this health challenge. Traumatic spondyloptosis represents the most severe and rare form of spinal injury, with subluxation of $>100 \%$ of adjacent vertebral bodies. In this injury, all 3 spinal columns are disrupted and show complete loss of alignment. Spondyloptosis usually results from high-velocity injury, ${ }^{5}$ and many patients will also have other associated injuries. Because we maintain a prospective database on all spinal cord injuries at our center, we decided to evaluate the treatments and their outcomes among patients with spondyloptosis in our local health care setting in which specialized rehabilitation facilities are lacking.

ABBREVIATIONS ASIA = American Spinal Injury Association; TLICS = thoracolumbar injury classification and severity.

SUBMITTED February 24, 2014. ACCEPTED October 23, 2014.

INCLUDE WHEN CITING Published online March 13, 2015; DOI: 10.3171/2014.10.SPINE1440.

DISCLOSURE The authors report no conflict of interest concerning the materials or methods used in this study or the findings specified in this paper. 


\section{Methods}

We retrospectively reviewed data in a prospectively maintained registry of spinal cord injuries at our center over 5 years (March 2008-March 2013). Records from all patients whose spinal deformity included a diagnosis of "listhesis" were reviewed to identify cases of spondyloptosis. Spondyloptosis was radiologically defined on CT scans of the spine as $>100 \%$ subluxation of the spinal column at 2 adjacent vertebral levels. All patients whose spinal deformity met the criterion of spondyloptosis were included in the study. The different types of spondyloptosis were further subclassified with the Denis classification. ${ }^{2}$ The clinical records for all the patients with spondyloptosis were also reviewed, and the grading of the American Spinal Injury Association (ASIA) was used to assess the severity of the spinal cord injuries. Observations on CT and MRI scans were used to calculate the scores of the thoracolumbar injury classification and severity (TLICS) scale for all patients. 9 Treatment (operative or conservative) of the patients was decided by the treating neurosurgeon. As a protocol in our center, all thoracolumbar spine instrumentation is performed with pedicle screws and rods with or without interbody cage placement. All patients were followed up clinically and radiologically via phone calls during the study.

\section{Results}

In total, our records review identified spondyloptosis in 20 patients ( 15 men, 2 boys, and 3 women) within the 5 -year period considered. The mean age of the patients was 27 years (range $12-45$ years). Seventeen patients (85\%) were 20-40 years old, and 3 patients (15\%) were younger than 20 years. All patients had injuries due to high-velocity trauma, most of which were due to a fall from height (9 patients [45\%]) or to road traffic accidents (7 patients [35\%]). Ten of the patients (50\%) had polytrauma. Four patients (20\%) had hemothorax, pneumothorax, or both; 2 patients (10\%) had an associated head injury; and 2 (10\%) had a retroperitoneal hematoma. A perinephric hematoma along with nephric laceration was observed in 1 patient $(5 \%)$, and 2 patients $(10 \%)$ had fractures at other spinal levels (Table 1).

The most common spinal level of injury was at the thoracolumbar junction, that is, from T-10 to L-2 (11 patients [55\%]). Seven patients (35\%) had spondyloptosis in the dorsal region (T1-9), and 1 patient (5\%) each had injuries at the lower lumbar and sacral vertebrae (Figs. 1-4).

In all patients, the severity of their spinal injuries was ASIA Grade A at the time of presentation. A patient with sacral spondyloptosis had a concomitant burst fracture at L-2 with compression of the conus, causing complete paraplegia. One patient with L3-4 lumbar spondyloptosis also had paraplegia. An MRI scan of the lumbar spine indicated that the spinal cord ended at L-4 in this patient (Fig. 4). The TLICS score was 8 (3 for morphology, 3 for posterior ligamentous complex, and 2 for neurological deficit) for all of the 19 patients operated on for spondyloptosis.

Thirteen patients (65\%) had a sagittal type of spondyloptosis, and 7 patients (35\%) had a coronal type. Of the 13 patients with sagittal spondyloptosis, 3 (23\%) had a flex- ion-dislocation type of fracture, and $10(77 \%)$ had a sheardislocation type of fracture. Shear injuries were further classified as posteroanterior type (6 patients [60\%]) and anteroposterior type (4 patients [40\%]). All patients with a coronal type of spondyloptosis had a flexion-rotation type of dislocation.

\section{Treatment and Timing of Surgery}

Nineteen $(95 \%)$ of the patients were treated surgically. The patient who had sacral spondyloptosis and paraplegia due to an associated L-2 burst fracture did not wish to undergo surgery for spondyloptosis and was operated on only for the L-2 burst fracture.

In patients with isolated spondyloptosis, surgery was performed within 48 hours of admission. In 14 patients who had polytrauma, the spine was operated on after stabilization of the other injuries. Thirteen patients $(65 \%)$ underwent surgery during Weeks 2 and 3 after admission. One patient with a severe head injury underwent a bifrontal decompressive craniotomy immediately after admission and underwent spinal fixation in Week 4 after admission.

\section{Surgery}

A posterior surgical approach was used for all 19 patients surgically treated for spondyloptosis. We performed a 4-level fixation (2 levels above and 2 levels below the lesion) with pedicle screws and a rod in all patients. Seven patients also underwent transpedicular corpectomy with cage placement.

Intraoperatively, we noted complete cord transection in 7 patients (35\%) and CSF leakage with a dural tear in 4 patients (20\%). The spinal cord was acutely bent in 8 patients $(40 \%)$ who underwent surgery for spondyloptosis. In cases of complete cord transection, the spinal cord was ligated with a purse string suture and sealed with fibrin glue to prevent postoperative CSF leakage. In all patients, the operative wound was closed in multilayer steps, paying special attention to the thoracolumbar fascia. Complete reduction of listhesis was achieved in $15(79 \%)$ of the patients surgically treated for spondyloptosis (Figs. 1-4). In 4 patients (21\%), a complete reduction of the listhesis could not be achieved; the residual listhesis was Grade I in 2 of these patients (10.5\%) and Grade II in the other 2 patients $(10.5 \%)$.

\section{Postoperative Complications}

One patient (5.2\%) operated on for spondyloptosis had CSF leakage from the wound site postoperatively. Four patients (21\%) developed pneumonia, and $2(10.5 \%)$ developed septicemia that prolonged the hospital stay. None of the patients developed bedsores (decubitus ulcers) or deep vein thrombosis during their hospital stay.

\section{Follow-Up Events}

The mean length of hospital stay was 20 days (range 8-36 days). All of the patients were discharged from the hospital for home-based rehabilitation. The mean length of the follow-up period was 37.5 months (range 3-60 months). Because the follow-up was conducted over the 


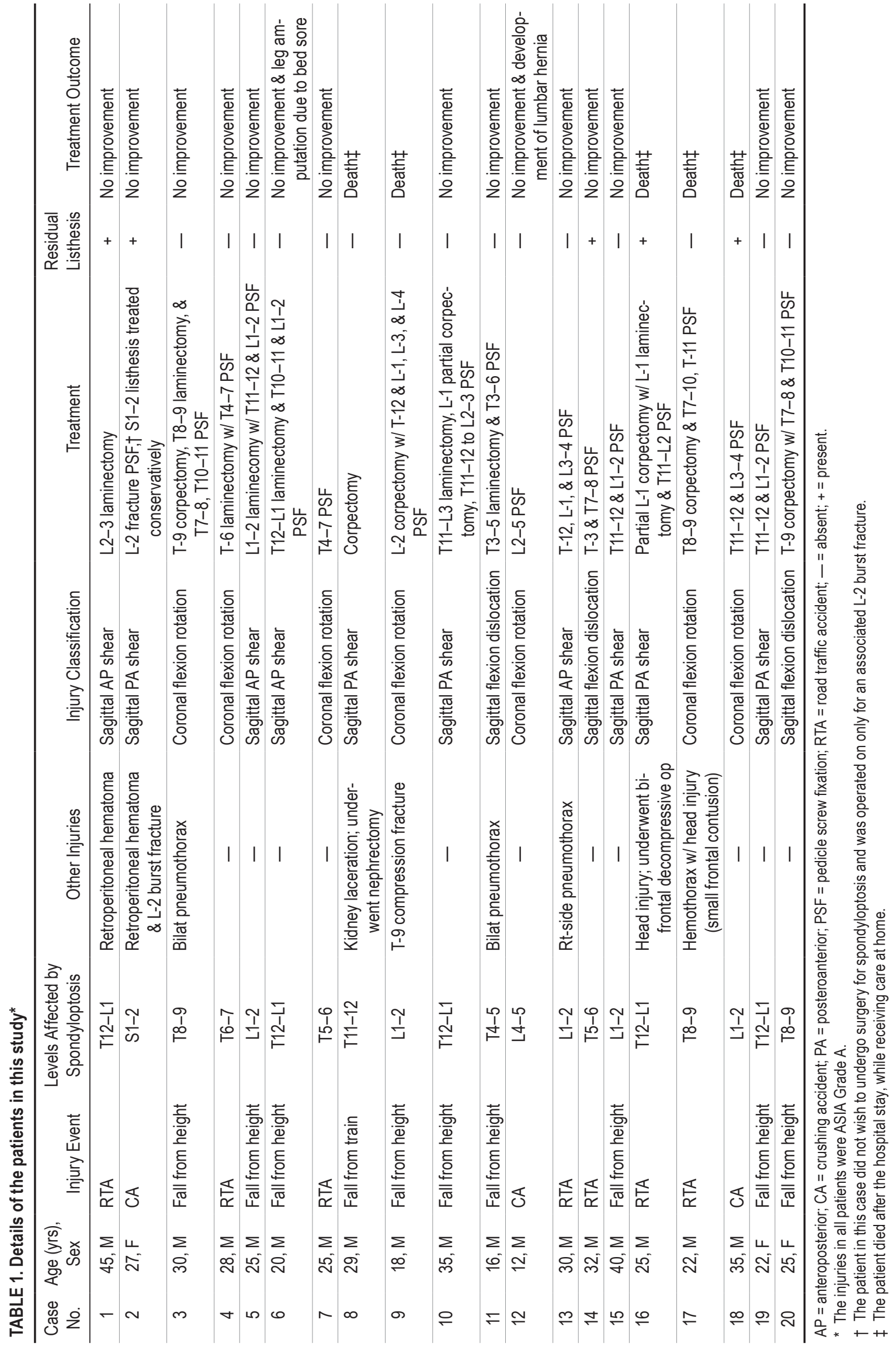



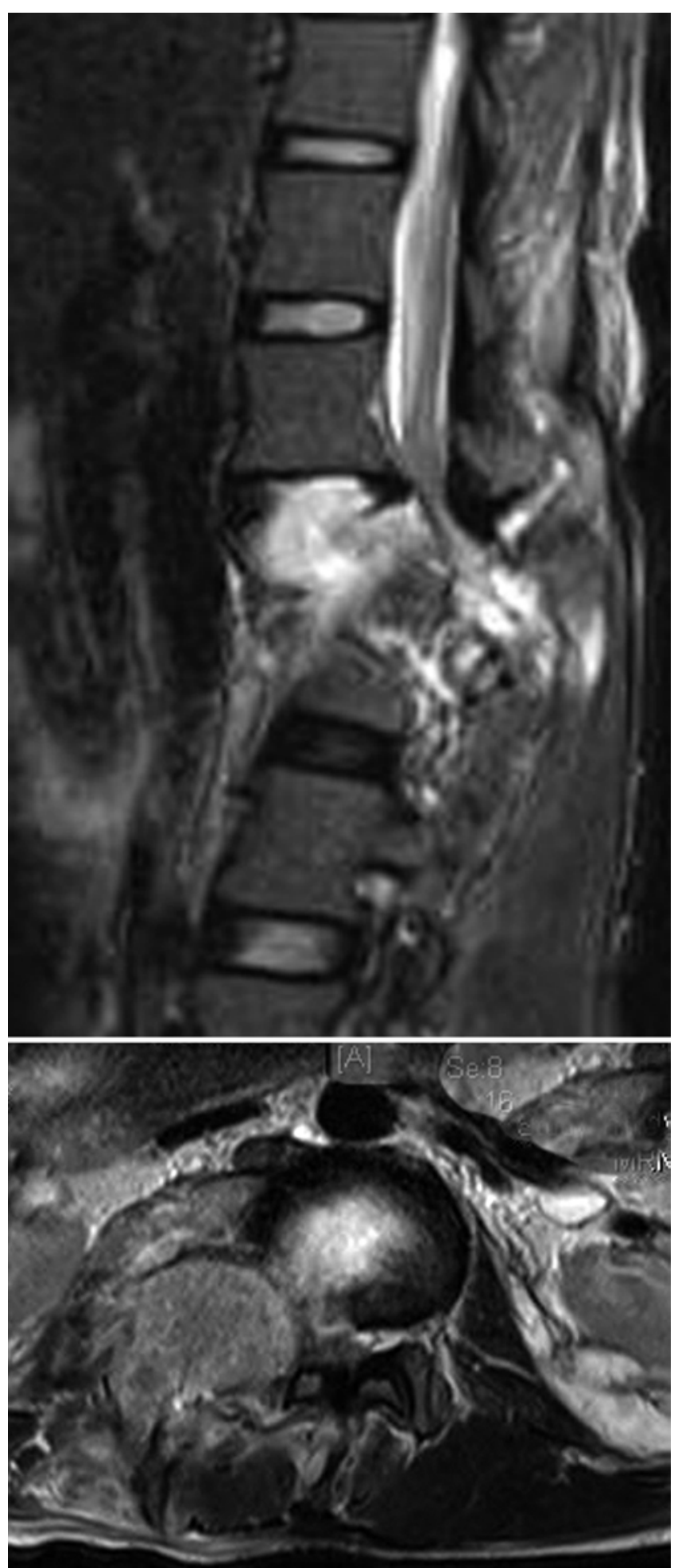

FIG. 1. Preoperative MR images of the lumbosacral spine, sagittal (upper) and axial (lower) views, showing a coronal spondyloptosis.

phone, radiographs were not available to assess fusion or pseudarthrosis. In total, 5 patients $(25 \%)$ died during the follow-up period, mainly from complications resulting from bed sores. One patient underwent amputation of the left lower limb below the hip joint after developing a large

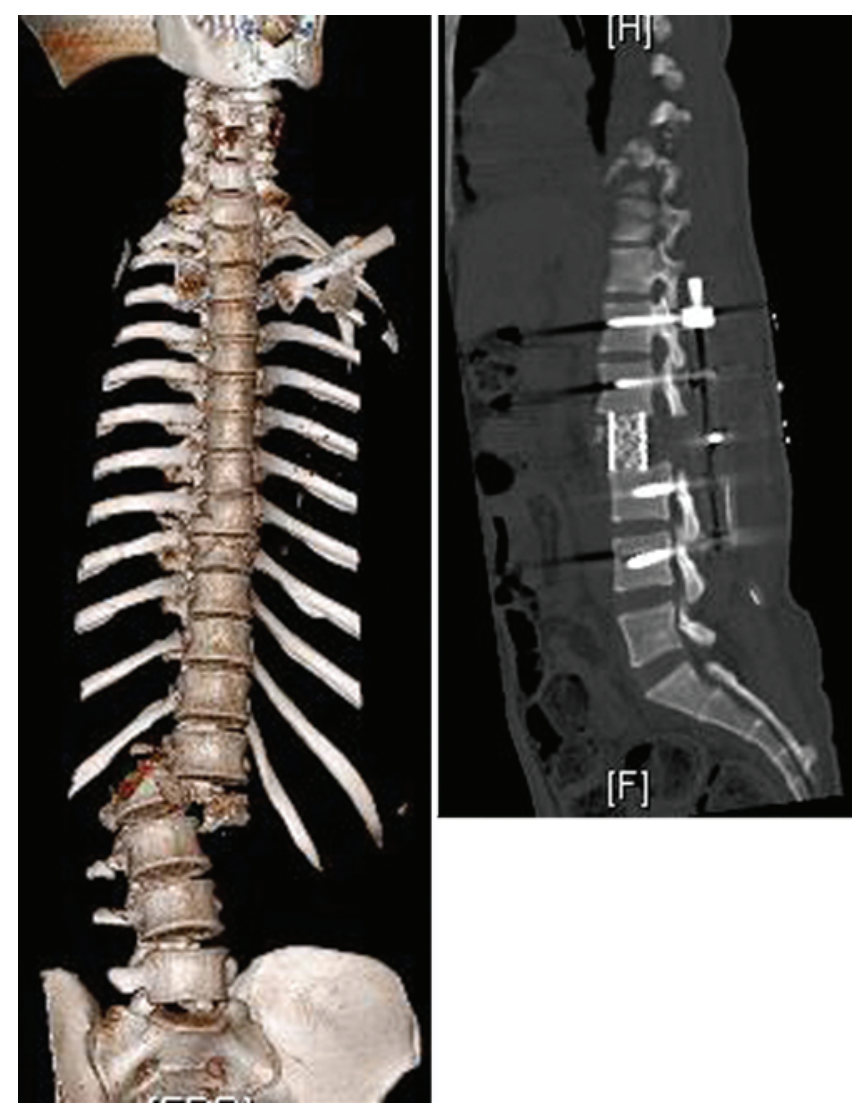

FIG. 2. Preoperative 3D CT image of the lumbosacral spine (left) and postoperative sagittal image (right) of the same patient showing good reduction of the spondyloptosis. Figure is available in color online only.

sacral bed sore. Another patient developed right-side lumbar hernia because of a weakened abdominal wall. This patient underwent herniorrhaphy in general surgery in the follow-up period. ASIA grades did not improve in any of the patients during the follow-up period.

\section{Discussion}

Spondyloptosis has been defined as listhesis of adjoining vertebrae of $\geq 100 \%$. ${ }^{6}$ It has been categorized as fracture dislocation in the Denis classification of spinal fracture $^{2}$ and is considered a very unstable injury involving all 3 sections of the spinal column. In $80 \%$ of cases, spondyloptosis is usually associated with complete neurological deficit. ${ }^{8}$ In 2007, Sekhon et al. described 2 cases of traumatic thoracic spondyloptosis with complete paraplegia. ${ }^{7}$ Both patients were operated on within $48-72$ hours of admission for spinal reduction and stabilization by a posterior route. Gitelman et al. reported a case of T6-7 spondyloptosis with completely preserved neurological functions and reviewed 22 cases of thoracic fracture dislocation without neurological deficit reported in the literature. ${ }^{3}$ The authors did not attempt reduction to preserve the spinal canal and instead performed in situ fusion.

To the best of our knowledge, the present study is the largest case series in the literature on traumatic spondyloptosis. The largest previous series was described by Yadla et al. ${ }^{10}$ and involved 5 patients with spondyloptosis 


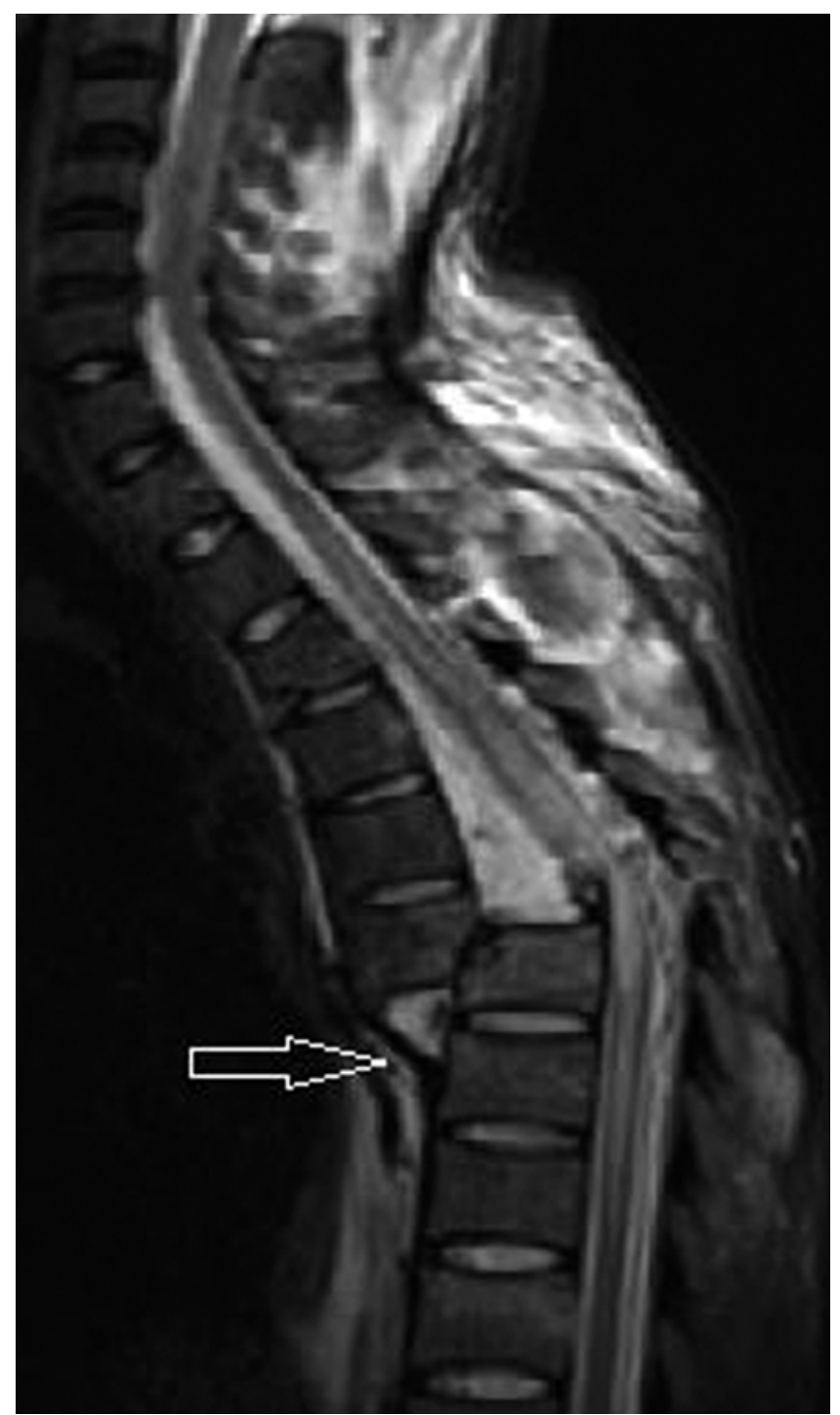

FIG. 3. Sagittal T2-weighted MR image of the dorsal spine showing sagittal spondyloptosis (of flexion-dislocation type). The arrow points to the stripped anterior longitudinal ligament.

of the thoracolumbar region. In that series, only 1 patient with an incomplete neurological deficit had an injury severity of ASIA Grade $\mathrm{C}$ and had spondyloptosis at Levels L1-2. The remaining patients had injuries above L-1 and their injury severity was ASIA Grade A at the time of admission. In our study, the injury severities in all patients were ASIA Grade $\mathrm{A}$ at the time of admission.

Case reports of L5-S1 spondyloptosis without neurological deficit have been reported in the literature. ${ }^{4}$ In our study, 1 patient who had sacral spondyloptosis had paraplegia due to an associated fracture at the L-2 level. In another patient with L3-4 spondyloptosis, preoperative MRI scans showed a low-lying cord, which may have been the cause of complete paraplegia in this patient.

In our study, the most common site of injury was the thoracolumbar spine (in 11 patients [55\%]). Such injuries are mainly due to the mechanical transition zone between the rigid thoracic and more mobile lumbar spine seg-
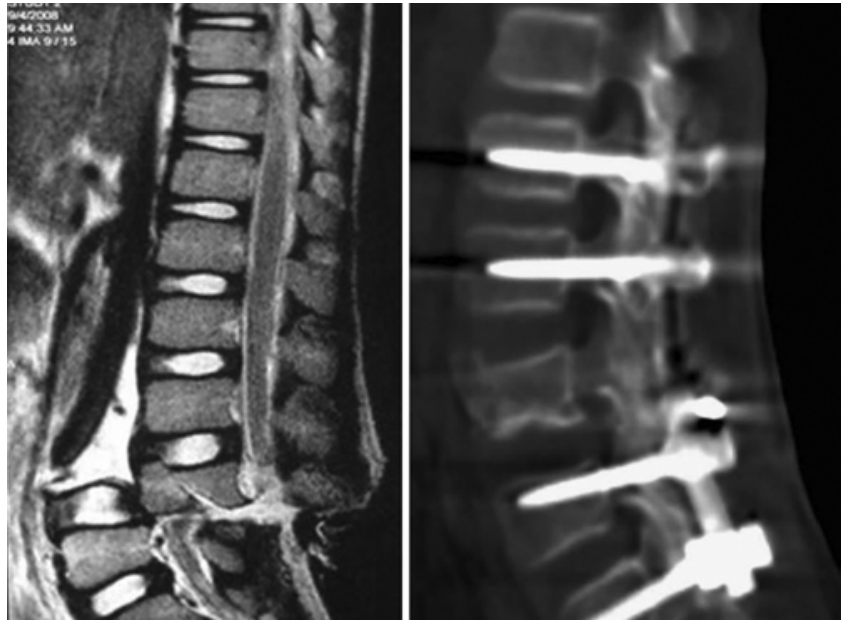

FIG. 4. Left: Preoperative sagittal T2-weighted MR image of the lumbar spine showing an L3-4 sagittal spondyloptosis (of shear type). This patient also had a low-lying cord. Right: Postoperative sagittal CT scan of the same region showing good spinal alignment after instrumentation.

ments. ${ }^{1}$ Seven patients $(35 \%)$ had injuries to the thoracic spine (T1-9) and 1 each (5\%) had spondyloptosis of the lower lumbar (L-2, L-3, and L-4) and sacral levels. These findings may be explained by the long segment of thoracic spine involved.

The types of spondyloptosis were sagittal (13 cases [65\%]) or coronal (7 cases [35\%]). We further categorized these cases with the Denis classification of spinal fracture. ${ }^{2}$ Denis classified fracture dislocations into the following 3 types: flexion dislocation, flexion rotation, and shear. The injury mechanism in flexion dislocation (which occurred in 3 of our patients) involves hyperflexion, which strips the anterior longitudinal ligament from the body of the lower vertebrae. A shear-type fracture involves hyperextension, and we further classified these fractures in our patients into posteroanterior (6 patients [60\%]) and anteroposterior (4 patients [40\%]). In the posteroanterior shear type, the superior segment moves in front of the inferior segment with resultant fracture of the posterior arch. This type of fracture is associated with a high incidence of spinal cord and dural injuries. In anteroposterior shear fractures, the inferior vertebrae move anteriorly to the upper segment, leaving the posterior arch intact. The coronal type of spondyloptosis involves flexion along with a rotation component and occurred in 7 of our patients (35\%).

All patients were operated on via the posterior route in a single stage. The aim of the surgery was to achieve the alignment of the vertebral column for early mobilization of the patients. After exposure of the region of interest and under an image intensifier, pedicle screws were placed 2 levels above and below the level of the fracture. Then, while maintaining sterile technique, abdominal pressure was applied by placing one hand below the abdomen (through sterile drapes). In our experience, this procedure relieves strain in the paraspinal muscles and either helps bring the vertebral column in complete alignment or restores much of the anatomy. This maneuver is more successful if surgery is undertaken the first few days after injury because a delay in surgery leads to inflammatory 
adhesion at the level of the disrupted joints with fibro-osseous fusion.

The next step of reduction required distraction of the vertebral column by placing a contoured rod on one side of the vertebral column. After reduction had been achieved, the rod was placed on the vertebral body of the opposite side and fixed with screws. The contoured rod, which was initially used for the distraction, was replaced by a straight rod. If distraction failed to bring about the spinal alignment, a transpedicular corpectomy was done. However, despite these maneuvers, complete alignment could not be achieved in 4 of the 19 patients (21\%) operated on for spondyloptosis. In their review of 22 cases of thoracic fracture dislocation, Gitelman et al. ${ }^{3}$ reported that complete alignment could not be achieved in $31.3 \%$ of their cases. However, even with a residual Grade II listhesis, we achieved our goal of vertebral column fixation and early mobilization of the patient. The thecal sac was ligated in 7 patients for complete cord transection and sealed with fibrin glue. We closed the wound in layers and paid special attention to the thoracolumbar fascia.

None of the patients died before being discharged from the hospital. As part of our standard protocol, we did not administer steroids to the patients, but complications due to infections were common during the hospital stay. We note that these complications were probably caused by the polytrauma in most of the patients. None of the patients developed deep vein thrombosis or bedsores during their hospital stay, which may reflect the high quality of our nursing care at our center and the strict implementation of a deep vein thrombosis prophylaxis protocol, consisting of low-MW heparin and graduated pneumatic stockings. In none of the patients, neurological function had recovered at the time of hospital discharge. We followed up the patients' neurological recovery and other outcomes and observed that none of the patients showed an improvement in strength during the follow-up period. We note that some case reports have observed neurological improvement after spondyloptosis correction. ${ }^{3,8,10}$ However, in all of these cases, the injury severity was ASIA Grade $\mathrm{C}$ or better at the time of presentation.

Five patients $(25 \%)$ died during the follow-up period. Lack of access to rehabilitation facilities, leading to bedsores while patients were being cared for at home, was the main reason for the observed mortality rate. One patient underwent amputation of the lower limb below the hip because of a malignant bedsore. The results of this study are sobering and suggest that home-based care is suboptimal for most patients with spondyloptosis. Although acute care for spinal cord injuries has improved, much remains to be done for improving rehabilitation facilities in developing countries.

\section{Conclusions}

This largest study of its kind on traumatic spondylop- tosis to date shows that almost all patients (that is, all patients in our study) with this condition will have complete injury. Rehabilitation is critical for ensuring that patients with this condition can live independently and in dignity. Lack of access to rehabilitation facilities is major cause of high rates of morbidity and mortality from traumatic spondyloptosis in developing countries.

\section{References}

1. Bohlman HH: Treatment of fractures and dislocations of the thoracic and lumbar spine. J Bone Joint Surg Am 67:165169,1985

2. Denis F: The three column spine and its significance in the classification of acute thoracolumbar spinal injuries. Spine (Phila Pa 1976) 8:817-831, 1983

3. Gitelman A, Most MJ, Stephen M: Traumatic thoracic spondyloptosis without neurologic deficit, and treatment with in situ fusion. Am J Orthop (Belle Mead, NJ) 38:E162-E165, 2009

4. Goni V, Gopinathan NR, Saini UC, Kantharajanna SB: Traumatic L5 over S1 spondyloptosis without neurological involvement managed nonoperatively: a case report. Chin J Traumatol 16:178-181, 2013

5. Meneghini RM, DeWald CJ: Traumatic posterior spondyloptosis at the lumbosacral junction. A case report. J Bone Joint Surg Am 85-A:346-350, 2003

6. Meyerding HW: Spondylolisthesis as an etiologic factor in backache. JAMA 111:1971-1976, 1938

7. Sekhon LH, Sears W, Lynch JJ: Surgical management of traumatic thoracic spondyloptosis: review of 2 cases. J Clin Neurosci 14:770-775, 2007

8. Shapiro S, Abel T, Rodgers RB: Traumatic thoracic spinal fracture dislocation with minimal or no cord injury. Report of four cases and review of the literature. J Neurosurg 96 (3 Suppl):333-337, 2002

9. Vaccaro AR, Lehman RA Jr, Hurlbert RJ, Anderson PA, Harris M, Hedlund R, et al: A new classification of thoracolumbar injuries: the importance of injury morphology, the integrity of the posterior ligamentous complex, and neurologic status. Spine (Phila Pa 1976) 30:2325-2333, 2005

10. Yadla S, Lebude B, Tender GC, Sharan AD, Harrop JS, Hilibrand AS, et al: Traumatic spondyloptosis of the thoracolumbar spine. J Neurosurg Spine 9:145-151, 2008

\begin{abstract}
Author Contributions
Conception and design: Agrawal. Acquisition of data: Agrawal, Mishra. Drafting the article: Mishra. Critically revising the article: Agrawal. Approved the final version of the manuscript on behalf of all authors: Agrawal. Administrative/technical/material support: Agrawal, Gupta, Sinha, Satyarthee, Singh. Study supervision: Agrawal.
\end{abstract}

\section{Correspondence}

Deepak Agrawal, Department of Neurosurgery, JPN Apex Trauma Center, All India Institute of Medical Sciences, Ansari Nagar, New Delhi 110029, India. email: drdeepak@gmail.com. 